\title{
Transfer of control system interface solutions from other domains to the thermal power industry
}

\author{
Bligård, L-O ${ }^{\mathrm{a}, *}$, Andersson, $\mathrm{J}^{\mathrm{a}}$ and Osvalder, A- $\mathrm{L}^{\mathrm{a}}$ \\ ${ }^{a}$ Department of Product and Production Development, Division Design \& Human Factors, \\ Chalmers University of Technology. SE-412 96 Gothenburg, Sweden.
}

\begin{abstract}
In a thermal power plant the operators' roles are to control and monitor the process to achieve efficient and safe production. To achieve this, the human-machine interfaces have a central part. The interfaces need to be updated and upgraded together with the technical functionality to maintain optimal operation. One way of achieving relevant updates is to study other domains and see how they have solved similar issues in their design solutions. The purpose of this paper is to present how interface design solution ideas can be transferred from domains with operator control to thermal power plants. In the study 15 domains were compared using a model for categorisation of human-machine systems. The result from the domain comparison showed that nuclear power, refinery and ship engine control were most similar to thermal power control. From the findings a basic interface structure and three specific display solutions were proposed for thermal power control: process parameter overview, plant overview, and feed water view. The systematic comparison of the properties of a human-machine system allowed interface designers to find suitable objects, structures and navigation logics in a range of domains that could be transferred to the thermal power domain.
\end{abstract}

Keywords: Thermal power plant, human-machine system, human-machine interface, graphical user interface

\section{Introduction}

In a thermal power plant the operators' roles are to control and monitor the process to achieve efficient and safe production. As the technical complexity increases with e.g. more use of sensors, higher level of automation, and higher demands for quality, the challenges of visualizing adequate process information for the operators increase as well [3]. A challenge for design of human-machine interfaces is that interface updates have to keep up with the change of complexity (e.g. increased use of automation) within the domain [2]. One way of find out relevant updates is to study other domains and understand how they have solved similar issues in their design solutions.

The purpose of this paper is to present how ideas from interface design solutions from some domains with operator control can be transferred to thermal power plants. This work is part of a larger research project concerning visualisation of process data with the operators' needs in focus, financed by the Thermal Engineering Research Institute (Värmeforsk) in Sweden. The overall purpose of the large project is to facilitate the plant to be able to run optimally for a longer period of time and thereby increase the plant's efficiency.

\section{Domain comparison}

In the study 15 different domains were compared using a framework for systemic categorisation of human-machine systems based on systems theory [4]. . The framework consists of four parts (basic system, control system, operator and organisation) with totally 23 system properties.

\footnotetext{
*Corresponding author. E-mail: lars-ola.bligard@chalmers.se
} 
- The basic system

- Number of elements to control

- Connection/impact between the elements

- Predict relations in the connections

- Dependence between the elements

- Impact by external factors

- Stability (capability to maintain structural)

- Speed of changes

- Potential hazard to environment and humans

- The control system

$\begin{array}{ll}\circ & \text { Data-collection } \\ \circ & \text { Analysis } \\ \circ & \text { Decision } \\ \circ & \text { Manipulation } \\ \circ & \text { Proximity to basic system } \\ \circ & \text { Data accuracy } \\ \circ & \text { Manipulation speed } \\ \circ & \text { Manipulation accuracy } \\ \circ & \text { Time of operator interact with control } \\ & \text { system }\end{array}$

- The operator
- Level general education
- Level specific education
- Level mental work

- The organisation
- Time in surveillance
- Level of team work
- Level of work rotation

The following domains were included in the study:

- thermal power

- nuclear power

- pulp and paper

- refinery

- $\quad$ ship engine control

- ship bridge

- aviation cock-pit

- air traffic control tower

- air traffic control area

- medical technology - intensive care

- surveillance centre

- emergency services centre

- computer game - first person shooter

- computer game - real time strategy

- computer game - sport manager

The categorisation of the domains made it possible to identify the domains which had properties comparable to the thermal power industry and thereby pos- sible to use for knowledge transfer regarding interface design solutions. The results from the comparison showed that the domains most suitable were:

- nuclear power

- refinery

- $\quad$ ship engine control

- medical technology - intensive care

- $\quad$ surveillance centre

- computer game - first person shooter

- computer game - real time strategy

\section{Useful interface solutions from the domains}

For the selected domains, objects, structures and navigation logics in the human-machine interfaces were studied deeper to elicit appropriate design solutions for operators in the thermal power domain. The focus was on graphical items presented on computer screens. The selection of ideas was made relying on the authors' expert knowledge and the main approach was to find solutions that could support thermal power plant operators in their work.

Below, the origin of the interface design used in a specific domain is briefly explained using the four parts of the framework. After that, a number of interesting interface solutions from the domains is listed.

\subsection{Nuclear power}

The interface design in nuclear power plant control rooms has a strong legacy from preceding designs. Also, the rigorous regulatory bodies have an indirect influence, since certain demands have to be fulfilled. In the nuclear power plant control rooms today, there is a hybrid mix of old and new technology, i.e. both old manual controls and gauges as well as computer based technology. Each control room is more or less a unique setting. The human-machine interfaces are mainly adapted to the technical system from a safety perspective.

\subsubsection{Design ideas from the nuclear power domain}

- A common frame of reference in the control room for all operators through use of a large screens showing overall status of the plant.

- Taking advantage of alarm displays that are designed to support quick diagnosis through pattern recognition.

- Most process values can be displayed as trends. 
- Live camera images show parts of the process in real time.

- Interfaces adapted for specific situations, e.g. emergency situations, start-up or shut-down manoeuvres.

- Filtering of expected alarms, e.g. expected low pressure alarms that always follow a pump stoppage are filtered.

\subsection{Refinery}

In the refinery domain, the interfaces design is dependent on which standard the control system developer is using.. Due to the large scale process there is a clear allocation of operator roles between different process sections. This is also reflected in the interface design where large screen displays are divided into adjacent sections making it easy to follow the process flow.

\subsubsection{Design ideas from the refinery domain}

- The interfaces are based on the technical process supporting the monitoring of the material flow in the refining process.

- Large screen displays are used to effectively overview large process sections - dark screens are used in normal conditions so that anomalies can be highlighted and easily detected.

\subsection{Ship engine control}

The reason for the interface design is mostly dependent on what the manufacturer is providing. The interface designs are adapted to the various parts of the technical system.

\subsubsection{Design ideas from the ship engine control domain}

- Function-based interfaces - there is a possibility to view all objects providing a specific function of the ship (e.g. pumps) on its own display.

- The operator can choose different interfaces using quick shortcuts.

- The operators can clearly see the process value in relation to the optimal state, making monitoring of important key parameters (e.g. fuel performance) easy.

\subsection{Medical technology - intensive care ventilator}

The main motivation behind the design of an intensive care ventilator is the adaptation to the intensive care context. The design is partly dependent on domain standards, but also on the manufacturing companies' own design solutions. The operator interface is mainly designed considering technological and measuring aspects, rather than the task to be performed by the operator.

\subsubsection{Design ideas from the medical technology domain}

- Use of curves and trends to display changes over time.

- Function-based interface - the interface is designed according to the functions in the equipment.

- Use of XY-plots for display of relationship between parameters.

- Possibility to silence audible alarms in advance (in some task the alarms are expected, but not disabled due to safety requirements)

\subsection{Surveillance centre}

In surveillance, the interfaces are adapted according to how the monitoring is performed in the specific surveillance centre. Adaptations are often made based on an existing platform developed by the supplier. The interfaces are mainly task based.

\subsubsection{Design ideas from the surveillance domain}

- Alarms are displayed graphically on maps.

- Status indication of objects (i.e. guards) are visible on the interface, often as a map over the surveillance area.

\subsection{Computer game - first person shooter}

In first person shooter computer games, an industry standard has been developed during the last 15 years. The design of the graphical user interface is well-adapted to the operator- a direct image of what is happening and the operator can control the game character directly. The most important information is always available. 
3.6.1. Design ideas from the computer game domain - first person shooter

- A scroll list with the latest events is shown

- Important information is always displayed irrespective of the screen image being used.

- Extended information is quickly accessible by pushing a button.

\subsection{Computer game - real time strategy}

In this domain the graphical user interface has gone through an evolutionary development based on earlier versions of the game and the standards that has been used within the genre. The interface is welladapted in relation to the controlled system (e.g. a battle field), the operator gets a direct image of what is happening. The interface is also well adapted to the control system, functions are easy to access and it is easy to follow the effects of actions. Since the operators do not have any previous knowledge of the specific game, the design makes quick learning possible.

\subsubsection{Design ideas from the computer game domain} - real time strategy

- A generic overview is displayed continuously displaying overall system status and specific object status.

- A faceplate is shown continuously displaying available actions for the selected object.

- The object selection is clearly indicated.

- Quick access to basic help functions is provided.

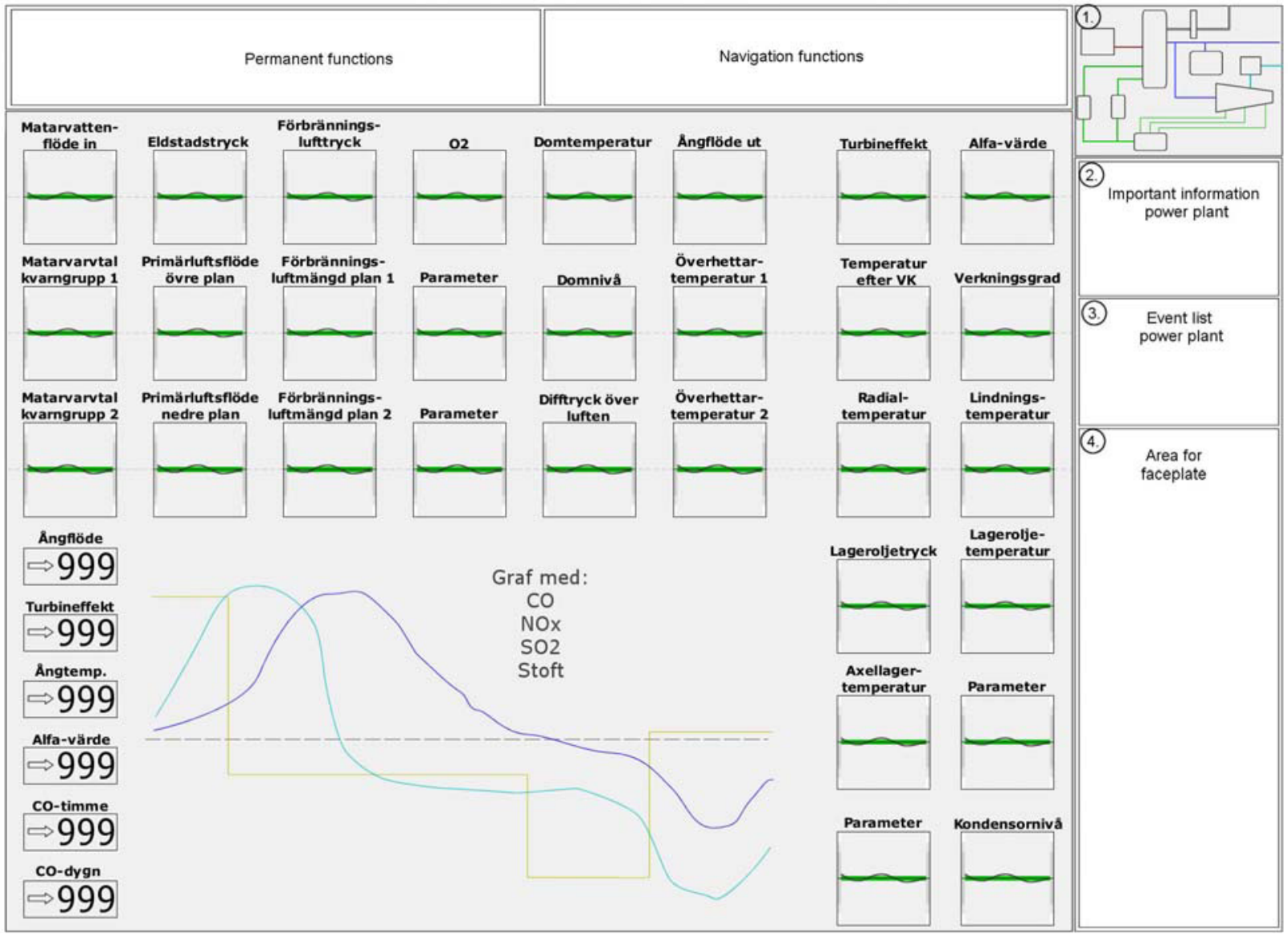

Figure 1 Interface solution for thermal power control: Process parameter overview 


\section{Conceptual interface solutions}

The useful interface solutions found from the studied domains were applied as a base for improvements of control system interface design for thermal power plants. One basic interface structure and three specific interface solutions were proposed for thermal power control: Process parameter overview (Figure 1), plant overview (Figure 2), and feed water view (Figure 3).

The basic interface structure which is common for all three proposals includes the following elements (indicated by numbers in Figure 1):

1. - A general overview is shown continuously

(Computer game - Real time strategy)

- Chosen objects are highlighted in the overview display (Figure 3) (Computer game - Real time strategy)

- Quick access in navigation from overview dis- play (Ship engine control)

2. Important information regarding the process is always visible (Computer game - First person shooter)

3. List of recent events (Computer game - First person shooter)

4. Dedicated place for faceplate (Computer game Real time strategy)

The interface solution for the process parameter overview (Figure 1) included the following transferred elements:

- Display for specific situations (Nuclear power)

- Common frame of reference (Nuclear power)

- Use of integrated trends of process values $(\mathrm{Nu}-$ clear power)

- Pattern recognition (Nuclear power)

- Overview displays of the entire facility (Refinery)

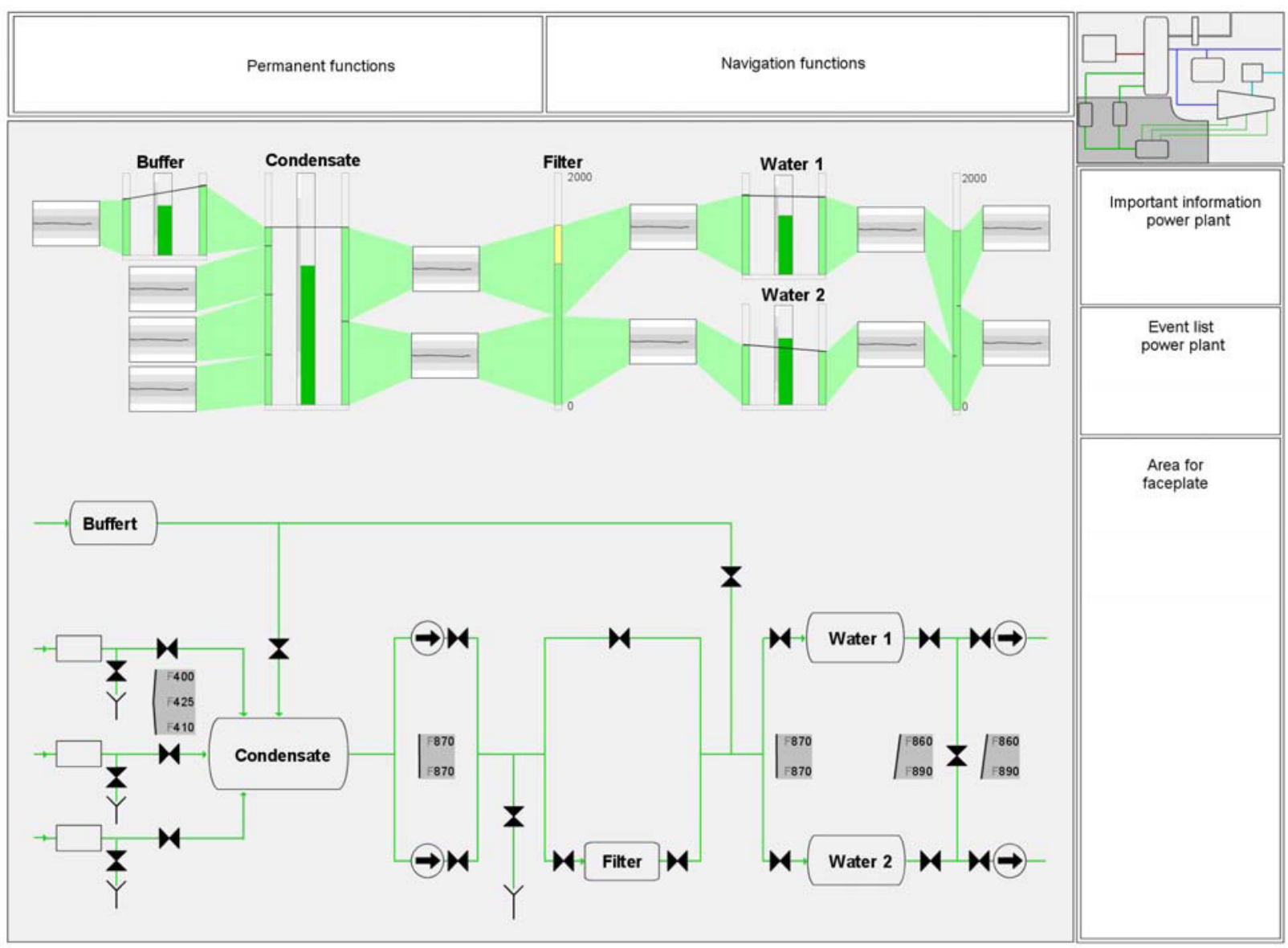

Figure 2 Interface solution for thermal power control: Plant overview 
- Monitoring of key process parameters (Ship engine control)

- The object status is visible directly in the interface (Surveillance centre)

The interface solution for the plant overview (Figure 2) included the following transferred elements:

- Display for specific situations (Nuclear power)

- Common frame of reference (Nuclear power)

- Pattern recognition (Nuclear power)

- Overview displays of the entire facility (Refinery)

- Monitoring of key process parameters (Ship engine control)

- Quick access in navigation from overview display (Ship engine control)

- Use of curves and trends (Medical technology Intensive care unit)
- Use of X-Y plots to describe the relationship between two parameters (Medical technology)

- Alarms are displayed on a map of the facility (Surveillance centre)

- The object status is visible directly in the interface (Surveillance centre)

The interface solution for the feed water system view (Figure 3) included the following transferred elements:

- Display for specific situations (Nuclear power)

- Display for improved understanding of system dynamics (Refinery)

- Use of curves and trends (Medical technology)

- Alarms are displayed on a map of the facility (Surveillance centre)

- The object status is visible directly in the interface (Surveillance centre)

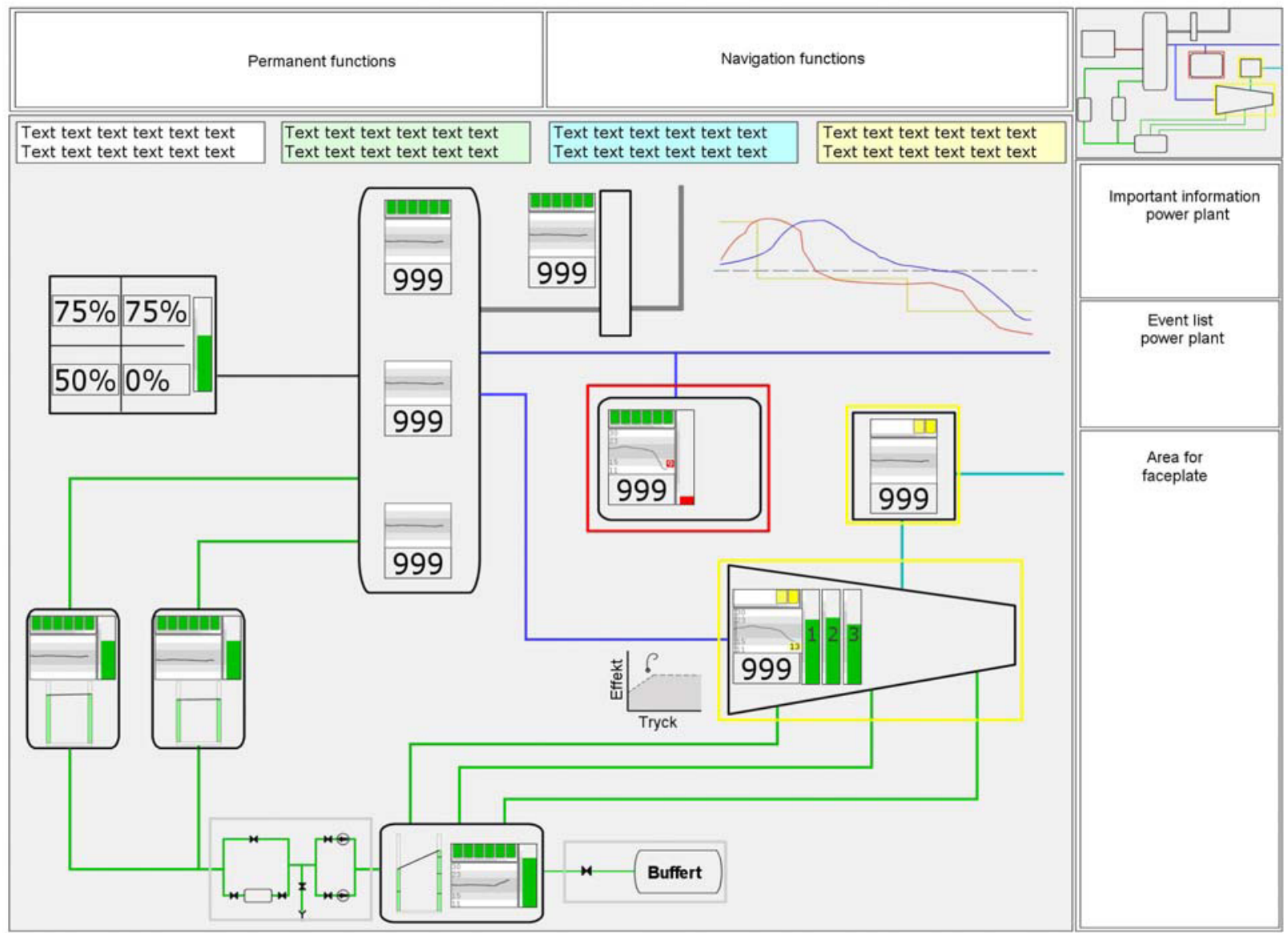

Figure 3 Interface solution for thermal power control: feed water view 
A more detailed description of the displays can be found in reference [1] and. [5].

\section{Discussion}

The framework for systematic comparison of the properties of a human-machine system enables the identification of similarities and differences between different domains. Using the framework is one way for interface designers to find suitable objects, structures and navigation logics in a range of domains that could be transferred to another domain. The comparison brings forward the possible benefit of using visualisations that are established as standard in other domains. Some of the ideas that were found in this study are already implemented in the thermal power domain.

In this study the selection of ideas to be used in the interface design was based on the authors' expert knowledge in the field of human factors engineering. The presented displays are only conceptual sketches of what a display might look like. However in a live industrial project, the investigation and specification of the operators' needs has to be done more thoroughly to meet the demands of a specific plant.

Five thermal power plant operators evaluated the developed display solutions presented here, using a power point mock-up, with a positive result [1]. They found the interface designs useful because their awareness of the process might be improved and the possibilities to work pro-actively would be enhanced compared to the control system they were using at the time of the evaluation.

By also studying domains that are too dissimilar to be of interest at a first sight, valuable ideas can be found. In this paper it has been shown that by using human-machine system properties, similarities and useful interface design ideas were found in domains dissimilar to thermal power. How a first-person shooter computer game can contribute to the design of an operator displays for a thermal power plant is not obvious, but with the systematic framework used in this paper interesting ideas were extracted. The systematic comparison can also be of help for bringing good ideas from one domain to another, and thus speeding up the design process. In this way it is possible to benefit from other engineers' or designers' work. The procedure may help the designer to 'go outside the box' by widening the inspirational view outside the specific domain in which the designer is working.
The drawback of a systematic working procedure is that it takes longer time to ensure quality. However a proficient designer can achieve new ideas without using a systematic approach, but the procedure is to some extent an assurance that no useful aspects are missed.

The work presented in this paper gives an example of how learning can take place between domains. It also shows how inspiration can be collected from domains that have great differences from the specific domain where the design work is performed. The systematic comparison can be used as a method for human factors engineers in industry to find new ways of presenting information by drawing inspiration from ideas already developed by others.

\section{References}

[1] L.O. Bligård, J. Andersson, A. Thunberg, and A.L. Osvalder, Graphical visualization of process status for thermal power plants, in Human Factors: A system view of human, technology and organisation, D. de Waard, Axelsson, A., Berglund, M., Peters, B. and Weikert, C., Editor. 2010, Maastricht, the Netherlands: Shaker Publishing.

[2] S. Guerlain, G.A. Jamieson, P. Bullemer, and R. Blair, The MPC elucidator: A case study in the design for humanautomation interaction. IEEE Transactions on Systems, Man, and Cybernetics Part A:Systems and Humans., 2002. 32(1): p. 25-40.

[3] J.D. Lee, Human Factors and Ergonomics in Automation Design, in Handbook of Human Factors and Ergonomics, G. Salvendy, Editor. 2006, John Wiley \& Sons, Inc.

[4] A. Osvalder, -L., L. Bligård, -O., J. Andersson, and A. Thunberg. Framework to describe and categorise a complex human-machine system. in Human Factors and Ergonomics Society Europe Chapter Annual Meeting. 2009. Linköping.

[5] A. Osvalder, -L., L. Bligård, -O., J. Andersson, and A. Thunberg. Framework to describe and categorise a complex human-machine system. in Human Factors: A system view of human, technology and organisation. 2010: Maastricht, the Netherlands: Shaker Publishing. 